\title{
Sinusoidal Movement of a Grating Across the Monkey's Fingerpad: Effect of Contact Angle and Force of the Grating on Afferent Fiber Responses
}

\author{
A. W. Goodwin and J. W. Morley \\ Department of Anatomy, University of Melbourne, Parkville, Victoria 3052, Australia
}

Responses were recorded from cutaneous afferents innervating mechanoreceptors in the monkey's fingerpad. When gratings of alternating grooves and ridges were moved sinusoidally back and forth across the receptive field, the responses of the afferent were often not equal for the 2 directions of movement. To investigate this phenomenon, the position of the center of the afferent's receptive field, relative to the contact area between the grating and the finger, was varied systematically. For some afferents, regardless of these relative positions, the response was always greater for a particular direction of movement. For other afferents, regardless of these relative positions, the responses for the $\mathbf{2}$ directions of movement were always equal. For a minority of afferents, the response was greater for movement in one particular direction for some relative positions and greater for movement in the opposite direction for other relative positions. Slowly adapting afferents (SAs), rapidly adapting afferents (RAs), and Pacinian afferents (PCs) exhibited all 3 types of response patterns. We could not relate these patterns to the afferent type or to the positions, in the fingerpad, of the receptive field center.

The contact force between the grating and the finger was varied by varying the contact displacement (indentation). Two grating spatial periods were used. For SAs and PCs the response increased with increasing indentation for both gratings. For RAs the response to the finer grating increased with increasing indentation, but the response to the coarser grating did not.

When a grating is moved back and forth over the fingertip, the responses of the mechanoreceptive afferents depend on both the spatial and the movement parameters of the grating (Goodwin and Morley, 1987; Morley and Goodwin, 1987). In addition, we have found that an afferent's response is not the same for the 2 directions of movement. In the tactile system, directionselective responses are a common feature of cortical neurons. They have been observed by many investigators (Schwartz and Fredrickson, 1971; Whitsel et al., 1972; Hyvärinen and Pora-

\footnotetext{
Received July 9, 1986; revised Dec. 9, 1986; accepted Jan. 8, 1987.

This work was supported by a grant from the National Health and Medical Research Council of Australia. We thank Neil Ratcliffe, Colin Clarke, and Alf Medoro for technical assistance, Kati Bromley for the illustrations, and Janet Bennett for typing the manuscript.

Correspondence should be addressed to Dr. A. W. Goodwin, Department of Anatomy, University of Melbourne, Grattan Street, Parkville, Victoria 3052, Australia.

Copyright (C) 1987 Society for Neuroscience $0270-6474 / 87 / 072192-11 \$ 02.00 / 0$
}

nen, 1978; Costanzo and Gardner, 1980; Iwamura et al., 1985), and the mechanism responsible for the selectivity is usually attributed to cortical circuitry. Many models of this circuitry are similar to the models of direction selectivity first proposed in the visual system, where the problem has been studied more extensively (Barlow and Levick, 1965; Gardner and Costanzo, 1980; Peterhans et al., 1985). When a monkey scans its fingertips over a grating, many of the cells in Brodmann's areas $3 \mathrm{~b}$ and 1 have asymmetric responscs, responding more vigorously to movement in one direction than to movement in the other direction (Darian-Smith et al., 1984). Darian-Smith et al. (1984) suggested that these asymmetries may, at least in part, be explained by a more effective engagement of laterally located receptive fields for one of the directions of movement. There has been an implicit assumption that the responses of primary afferents are symmetric: if a grating were moved back and forth over the center of a receptive field, then the afferent's response would be symmetric, that is, the same for both directions of movement. However, we have found that this is not always the case and, in this study, we have systematically investigated the nature of the asymmetry in the peripheral mechanoreceptive afferents.

From common experience it is clear that when you rub your finger back and forth over a textured surface, the sensation changes with changes in contact force between the finger and the surface. However, the psychophysical support for this perception is not clear-cut. Using scaling experiments, Lederman and Taylor (1972) have shown that the subjective estimate of the roughness of gratings increases with an increase in contact force, but that subjects are able to grade gratings of different roughness over a wide range of contact forces. Other studies have minimized the importance of contact force. For example, Lamb (1983) found that the ability to discriminate 2 dot patterns with different spacings between the dots was not dependent on contact force. Many studies (e.g., Morley et al., 1983) have not controlled the contact force but have allowed the subjects to select their own forces and thus make no comment on the effect of changes in force. Available neurophysiological data at the primary afferent level is even more equivocal. While the importance of contact force (or displacement) for punctate stimuli is well established (Lindblom and Lund, 1966; Mountcastle et al., 1966; Johnson, 1974; Knibestöl, 1975), the suggestion is that it is less important for textured surfaces. Johnson and Lamb (1981) found that the resolution of Braille-like dots by the mechanoreceptive afferent population was not significantly affected by a change in contact force. Using gratings moving over the fingertip at constant speed, Darian-Smith and Oke (1980) found 

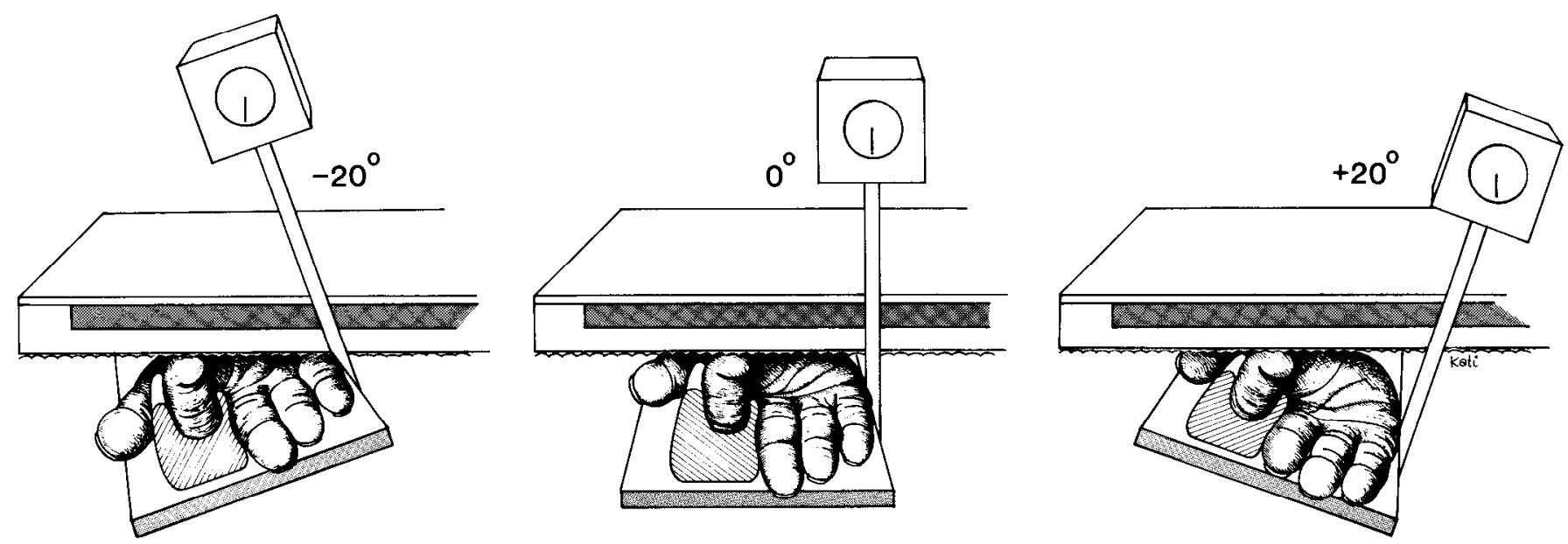

Figure 1. Changing the contact area between the grating and the finger. Center, Grating was tangential to the fingerpad at the receptive field center. The diagram has been stylized for clarity; actually, the finger was imbedded up to the nail in Plasticine (shaded cutaway) and the hand was taped to the Perspex plate, which could be raised or lowered by a micrometer (see Fig. 1 of Goodwin and Morley, 1987). Left, The hand was rotated by $20^{\circ}$ in a direction corresponding to supination $\left(-20^{\circ}\right)$. Right, The hand was rotated by $20^{\circ}$ in a direction corresponding to pronation $\left(+20^{\circ}\right)$. In all 3 cases, the micrometer raised or lowered the Perspex plate in a direction orthogonal to the grating. The angle of rotation was measured by the indicator attached to the plate.

little change in afferent responses with a 4-fold increase in contact force from 20 to 80 grams weight. Darian-Smith et al. (1980) found that the mean discharge rate of mechanorcceptors rcsponding to dot patterns generally increased with an increase in contact force, but they were unable to detail the effect from their data. However, none of the above studies varied contact force systematically. In the following experiments we have quantified the effect of changing contact force on the responses of the primary mechanoreceptive afferents.

\section{Materials and Methods}

As described previously (Goodwin and Morley, 1987), monkeys were anesthetized, and the responses of single mechanoreceptive afferent fibers, isolated by microdissection, were recorded. All slowly adapting afferents (SAs) and rapidly adapting afferents (RAs) studied had receptive fields located centrally on the fingerpad. For many of the Pacinian afferents (PCs) the receptive field could be localized to the distal phalanx and for the remainder the field could be localized to a particular digit. (A few of these remaining fields seemed localized to the 2 nd or 3 rd phalanges, but most could not be localized beyond the level of a particular digit.) Nylon gratings of alternating grooves and ridges were moved across the receptive fields with a sinusoidal motion. Some of the data for these results were obtained from the 22 SAs, $30 \mathrm{RAs}$, and 7 PCs described in the previous papers (Goodwin and Morley, 1987; Morley and Goodwin, 1987). The remaining data were collected from an additional 11 SAs, 7 RAs, and 6 PCs in $2 M$. nemestrina.

Response asymmetry. The center of the receptive field was estimated using a series of graded von Frey hairs and was marked with an ink dot. The fingerpad was photographed to show the exact position of the estimated receptive field center and its relationship to the skin ridges. As shown in Figure 1 (center) and in the first paper (Goodwin and Morley, 1987, Fig. 1), the stimulator holding the grating was oriented so that when the grating was lowered onto the fingertip, initial contact occurred at the center of the receptive field with the grating tangential to the fingerpad at that point: the axis of movement of the grating was orthogonal to the long axis of the finger. The finger was indented into the moving grating by a fixed distance from the point of contact (usually $1 \mathrm{~mm}$ ) and responses were recorded for a predetermined number of cycles, after which the finger was lowered clear of the grating. Then the hand was rotated by $10^{\circ}$ and the stimulation procedure repeated: The axis of rotation of the hand corresponded to the long axis of the finger (Fig. 1). This sequence was repeated until data had been collected at angles of rotation, in both directions, separated by $10^{\circ}$ over as large a range as possible. The usual total range of rotation was $80^{\circ}$, the limits being imposed either by one of the other fingers touching the grating or by the hand holder fouling the stimulator. The angle was defined as positive when the direction of rotation of the hand corresponded to pronation and negative when it corresponded to supination. During stimulation the computer recorded the responses of the afferent, the instantaneous position of the grating, and the instantaneous contact force between the grating and the finger.

For each afferent class, 2 combinations of stimulus parameters were used. For the SAs, a grating with a spatial period of $3 \mathrm{~mm}$ moving with a peak speed of $60 \mathrm{~mm} / \mathrm{sec}$ and a grating with a spatial period of $2 \mathrm{~mm}$ moving with a peak speed of $40 \mathrm{~mm} / \mathrm{sec}$ were used: in both cases the peak temporal frequency of the ridges was $20 \mathrm{~Hz}$. For the RAs the spatial periods of the 2 gratings were 2 and $1 \mathrm{~mm}$ moving with peak speeds of 80 and $40 \mathrm{~mm} / \mathrm{sec}$ respectively, resulting in a peak temporal frequency of the ridges of $40 \mathrm{~Hz}$. The 2 gratings used for the PCs had spatial periods of 2 and $1 \mathrm{~mm}$ moving at peak speeds of 160 and 80 $\mathrm{mm} / \mathrm{sec}$, respectively, resulting in a peak temporal frequency of the ridges of $80 \mathrm{~Hz}$. The standard amplitude of indentation of the finger into the grating was $1 \mathrm{~mm}$ from the point of contact. Sometimes additional data were collected with other values of the stimulus parameters.

The contact area between the grating and the finger, at the various angles of rotation, was estimated by fingerprinting as follows. The stimulator was stopped, and the grating was replaced by a piece of white paper mounted on a steel backing. The fingerpad was inked and indented into the paper by the same amount as used in the data collection (usually $1 \mathrm{~mm}$ from contact). The resulting static contact force was measured via the strain gauge bridge. This process was repeated for all angles of rotation at which data were collected.

For the PC afferents it was not possible to define the center of the receptive field with any precision, nor was it usually possible to locate the grating tangential to the finger at such a point. Thus, with PCs the strategy adopted was to proceed as if the receptive field center coincided with the center of the fingerpad, the results being interpreted accordingly.

Varying indentation amplitude. In this series of experiments the amplitude of indentation, from the point of contact, was varied systematically. The grating was positioned so that it contacted the center of the receptive field tangential to the fingerpad. Data were collected with indentation amplitudes of $0.5,0.75,1,1.25 \mathrm{~mm}$, and, occasionally, 0.25 or $1.5 \mathrm{~mm}$. Contact force between the finger and the grating was measured by a strain gauge with a resolution of $0.0025 \mathrm{~N}$, low-pass filtered with a corner frequency of $2 \mathrm{~Hz}$. Photographs recorded the position of the receptive field center on the fingerpad and its relation to the skin ridges. Fingerprints were used to estimate the contact area at the various indentation amplitudes. The same combinations of grating spatial periods, peak movement speeds, and peak temporal frequencies of the ridges were used as in the rotation experiments. For some fibers, in- 


\begin{tabular}{|c|c|c|c|c|c|c|c|}
\hline \multirow{2}{*}{$\begin{array}{l}\text { Fre- } \\
\text { quency }\end{array}$} & \multicolumn{7}{|c|}{ Spatial period (mm) } \\
\hline & 0.75 & 1.0 & 1.25 & 1.5 & 1.75 & 2.0 & 3.0 \\
\hline \multicolumn{8}{|l|}{$10 \mathrm{~Hz}$} \\
\hline$\mu$ & & & & & & 0.428 & 0.231 \\
\hline$\sigma$ & & & & & & 0.265 & 0.180 \\
\hline$n$ & & & & & & 8 & 18 \\
\hline \multicolumn{8}{|l|}{$20 \mathrm{~Hz}$} \\
\hline$\mu$ & & 0.362 & 0.426 & 0.357 & 0.341 & 0.276 & 0.169 \\
\hline$\sigma$ & & 0.350 & 0.281 & 0.293 & 0.201 & 0.219 & 0.122 \\
\hline$n$ & & 5 & 4 & 13 & 9 & 27 & 29 \\
\hline \multicolumn{8}{|l|}{$40 \mathrm{~Hz}$} \\
\hline$\mu$ & 0.711 & 0.480 & 0.568 & 0.386 & 0.374 & 0.277 & 0.241 \\
\hline$\sigma$ & 0.357 & 0.366 & 0.295 & 0.299 & 0.258 & 0.225 & 0.215 \\
\hline$n$ & 3 & 6 & 6 & 10 & 9 & 15 & 19 \\
\hline \multicolumn{8}{|l|}{$80 \mathrm{~Hz}$} \\
\hline$\mu$ & & 0.560 & 0.595 & 0.407 & 0.414 & 0.396 & 0.295 \\
\hline$\sigma$ & & 0.366 & 0.321 & 0.342 & 0.317 & 0.288 & 0.254 \\
\hline$n$ & & 5 & 6 & 8 & 8 & 13 & 18 \\
\hline \multicolumn{8}{|l|}{$160 \mathrm{~Hz}$} \\
\hline$\mu$ & & & 0.727 & 0.538 & 0.482 & 0.458 & 0.354 \\
\hline$\sigma$ & & & 0.272 & 0.316 & 0.298 & 0.282 & 0.206 \\
\hline$n$ & & & 4 & 6 & 6 & 10 & 10 \\
\hline
\end{tabular}

Columns in the table correspond to the spatial period of the grating and rows to the peak temporal frequency of the grating ridges. For each combination, $n$ indicates the number of afferents sampled; $\mu$, the average value of the contrast; and $\sigma$, the SD. Contrast is defined as $\left|R_{1}-R_{2}\right| /\left(R_{1}+R_{2}\right)$, where $R_{1}$ and $R$, are the responses to the 2 directions of movement.

dentation amplitudes of 1 and $1.25 \mathrm{~mm}$ were compared for a wide range of grating spatial periods and peak movement speeds. Occasionally, other combinations of parameters were also used.

\section{Results}

\section{Asymmetry of responses}

When a grating moved back and forth across the receptive field, the afferent usually responded better when the grating moved in one direction than when it moved in the opposite direction. This is illustrated by the cycle histogram in Figure 2 (bottom row, center column) for an $\mathrm{SA}$ responding to a $3 \mathrm{~mm}$ grating moving sinusoidally over the receptive field with a peak speed of $60 \mathrm{~mm} / \mathrm{sec}$ and thus with a resulting peak temporal frequency of the ridges of $20 \mathrm{~Hz}$. The response in the first half of the histogram, when the surface moved over the fingerpad in the ulnar direction, was less than the response in the second half of the cycle, when the surface moved over the fingerpad in the radial direction: the ratio of the mean responses over the 2 half cycles was 1.7. The center of the SA's receptive field was located as accurately as possible using a series of graded von Frey hairs applied by hand and was estimated to be at the center of the fingerpad (dot in Fig. 2, top row, center column). As explained in Materials and Methods, the grating was oriented so that when the finger was raised to contact the grating, initial contact occurred at the center of the receptive field with the grating tangential to the skin. Thus, there was no a priori reason to expect asymmetry in the responses to the 2 directions of grating movement.

All the SAs and RAs we studied had receptive fields located on the central portion of the fingerpad. Their estimated receptive field centers did not always coincide with the exact center of the fingerpad, but were always within a few millimeters of it, so that no receptive fields were anywhere near interphalangeal joints or nail beds or located on the "sides" or "end" of the fingertip. In all cases the grating was oriented tangential to the fingerpad at the receptive field center. Nevertheless, only a minority of afferents showed symmetric cycle histograms with responses equal for the 2 directions of grating movement. The degree of asymmetry in different afferents varied from only slight to so marked that the afferent effectively responded to only a single direction of motion.

To illustrate the range of asymmetry seen in our sample of afferents, a convenient measure is the response contrast. This is defined as $\left|R_{1}-R_{2}\right| /\left(R_{1}+R_{2}\right)$, where $R_{1}$ and $R_{2}$ are the responses to the 2 directions of movement. Thus, contrasts range from 0 (symmetric) to 1 (response in one direction only). This measure is used in preference to a ratio of the 2 responses to avoid the problem of excessively high (or even infinite) ratios as one of the responses becomes very small (or zero). Tables 1 and 2 show the contrasts for the SAs and RAs, respectively. Each entry corresponds to a different combination of stimulus parameters and shows the mean value and SD of the contrast, and the number of afferents sampled for that combination of parameters.

For the PCs the experimental protocol was slightly different. All PCs we studied had receptive fields that could be confidently localized to a single finger. For many PCs, the fields could not be localized further than that with any confidence, while for some the field could be further localized to the fingerpad or the second phalanx, etc. Thus for all Pacinians we stimulated the finger as if the center of the receptive field were located at the center of the fingerpad and interpreted the results accordingly 

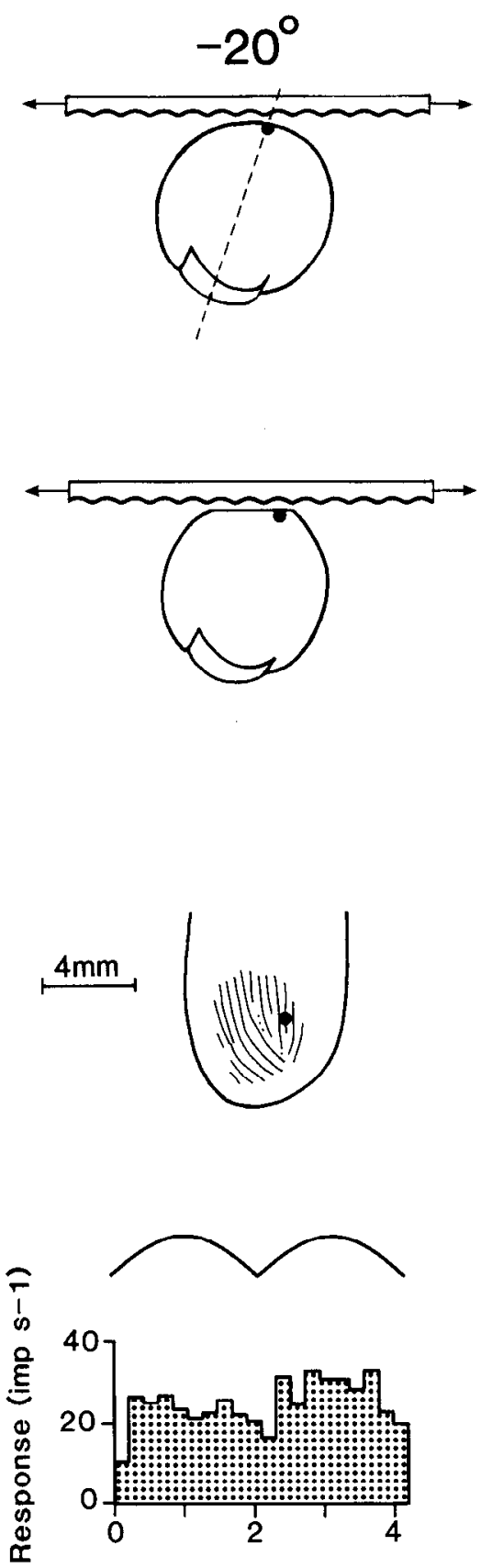
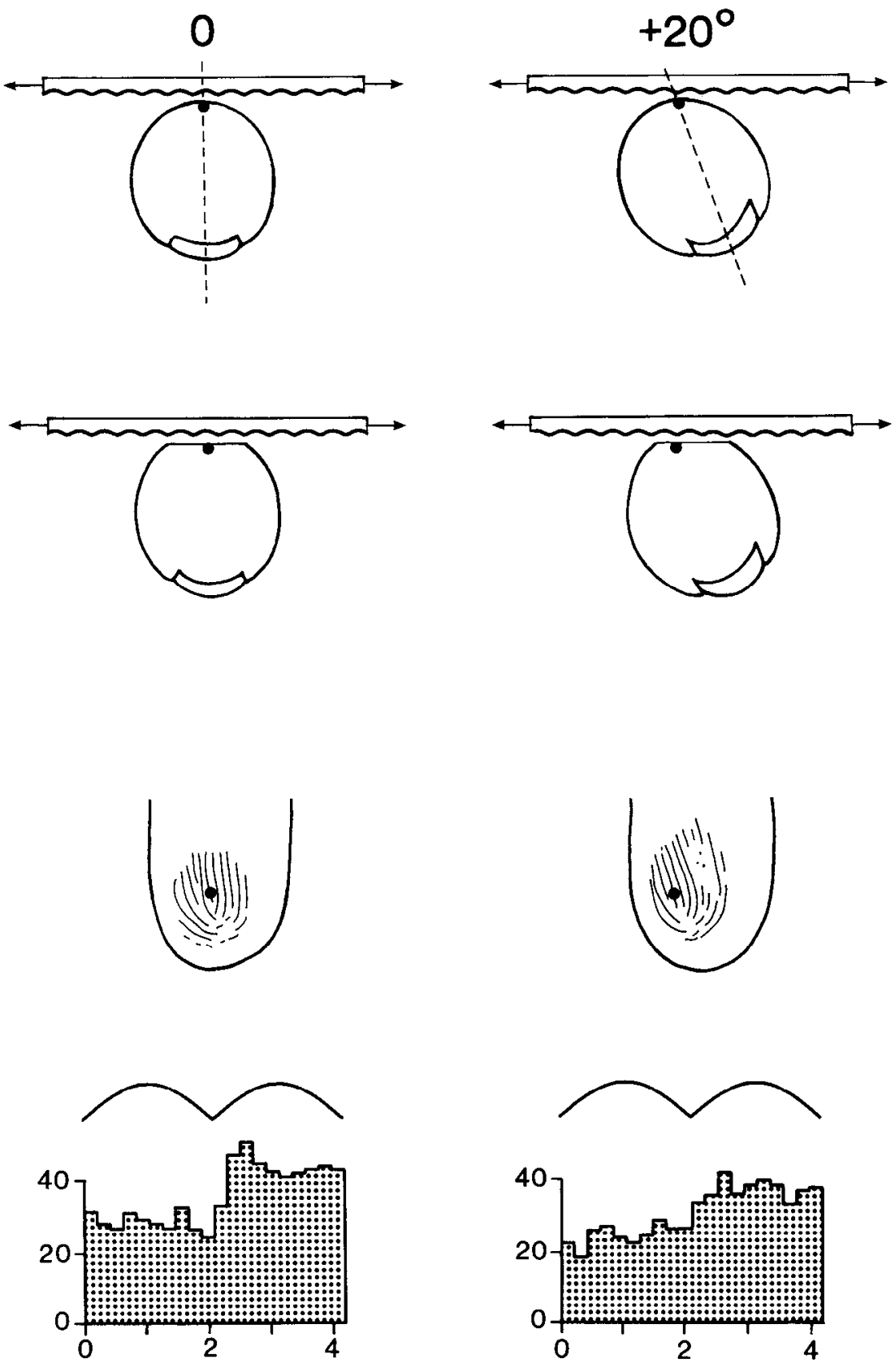

Time (s)

Figure 2. Responses of an SA, at 3 angles of hand rotation, to a $3 \mathrm{~mm}$ grating moving at a peak speed of $60 \mathrm{~mm} / \mathrm{sec}$ (peak temporal frequency of the grating ridges, $20 \mathrm{~Hz}$ ). The receptive field center (RFC) of the SA was located at the center of the fingerpad. Going down the center column, the top row shows an end view of the finger, with the RFC indicated by a dot, immediately prior to contact with the moving grating, which was tangential to the fingerpad at the RFC. The second row shows an end view of the finger after it had been indented $1 \mathrm{~mm}$ into the grating; the RFC was at the center of the contact area between the finger and the grating. The third row shows a top (ventral) view of the finger; lines show the pattern of skin ridges in the contact oval, with the RFC located at the center of the oval. The bottom row shows the cycle histogram of the afferent's responses averaged over 17 cycles (bin width $0.209 \mathrm{sec}$ ): During the first half cycle the grating moved in the ulnar direction and during the second half cycle in the radial direction. The left column shows the same data when the finger was rotated, about its long axis, by $20^{\circ}$ in a direction corresponding to supination $\left(-20^{\circ}\right)$ and the right column when the finger was rotated, about its long axis, by $20^{\circ}$ in a direction corresponding to pronation $\left(+20^{\circ}\right)$.

(see Discussion). Like SAs and RAs, the PCs showed a rangc of asymmetry in their responses to the 2 directions of movement, as shown in Table 3.

As can be seen in Tables 1-3, there was a wide range of contrasts for all 3 afferents. Before running the gratings over the receptive field of a particular afferent, we could not predict how asymmetric the responses would be, even though we knew the cstimatcd position of the receptive ficld center. The asymmetry was not influenced by the initial direction of the grating motion.

\section{Finger rotation}

Is it possible that the response asymmetries discussed above resulted from errors in estimating the receptive field center? The use of hand-held von Frey hairs is, to some extent, subjective. 


\begin{tabular}{|c|c|c|c|c|c|c|c|}
\hline \multirow{2}{*}{$\begin{array}{l}\text { Fre- } \\
\text { quency }\end{array}$} & \multicolumn{7}{|c|}{ Spatial period (mm) } \\
\hline & 0.75 & 1.0 & 1.25 & 1.5 & 1.75 & 2.0 & 3.0 \\
\hline \multicolumn{8}{|l|}{$10 \mathrm{~Hz}$} \\
\hline$\mu$ & & & & & & 0.067 & 0.043 \\
\hline$\sigma$ & & & & & & 0.087 & 0.032 \\
\hline$n$ & & & & & & 3 & 12 \\
\hline \multicolumn{8}{|l|}{$20 \mathrm{H} z$} \\
\hline$\mu$ & & & & 0.153 & 0.075 & 0.079 & 0.053 \\
\hline$\sigma$ & & & & 0.118 & 0.083 & 0.081 & 0.055 \\
\hline$n$ & & & & 14 & 9 & 16 & 17 \\
\hline \multicolumn{8}{|l|}{$40 \mathrm{~Hz}$} \\
\hline$\mu$ & 0.166 & 0.145 & 0.130 & 0.131 & 0.065 & 0.065 & 0.046 \\
\hline$\sigma$ & 0.150 & 0.140 & 0.100 & 0.142 & 0.059 & 0.054 & 0.037 \\
\hline$n$ & 17 & 34 & 10 & 26 & 9 & 31 & 20 \\
\hline \multicolumn{8}{|l|}{$80 \mathrm{~Hz}$} \\
\hline$\mu$ & 0.190 & 0.182 & 0.175 & 0.135 & 0.087 & 0.076 & 0.054 \\
\hline$\sigma$ & 0.158 & 0.123 & 0.120 & 0.131 & 0.075 & 0.077 & 0.044 \\
\hline$n$ & 21 & 18 & 11 & 20 & 7 & 18 & 18 \\
\hline \multicolumn{8}{|l|}{$160 \mathrm{~Hz}$} \\
\hline$\mu$ & 0.250 & 0.278 & 0.200 & 0.156 & 0.136 & 0.108 & 0.086 \\
\hline$\sigma$ & 0.158 & 0.153 & 0.103 & 0.145 & 0.144 & 0.091 & 0.077 \\
\hline$n$ & 20 & 16 & 10 & 17 & 9 & 18 & 17 \\
\hline \multicolumn{8}{|l|}{$320 \mathrm{~Hz}$} \\
\hline$\mu$ & 0.224 & 0.254 & 0.155 & 0.148 & & & \\
\hline$\sigma$ & 0.162 & 0.130 & 0.158 & 0.115 & & & \\
\hline$n$ & 12 & 13 & 6 & 13 & & & \\
\hline
\end{tabular}

Format as in Table 1 .

Moreover, this method only allows an estimate of the point in the receptive field most sensitive to steplike indentation by punctate stimuli. This may not be an appropriate reference point for gratings moving over the skin. To answer this question the contact between the skin and the grating was varied systematically as shown in Figure 2.

The top row, center column of Figure 2 shows an end view of a monkey's finger. An SA afferent had a receptive field center, shown by the dot, located at the center of the fingerpad. When the finger was raised to contact the moving grating, initial contact occurred at the receptive field center. Indenting the finger into the grating, by raising it an additional $1 \mathrm{~mm}$, "flattened" the fingerpad as shown. The receptive field center was located in the middle of the resulting contact area. The response of the afferent to sinusoidal movement of the grating can be seen in the cycle histogram in the bottom row, center column. This histogram is obviously asymmetric, with the afferent responding less vigorously when the grating moved in the ulnar direction (first half cycle) than when it moved in the radial direction (second half cycle). The ratio of the mean responses for the 2 half cycles was 1.5 and the contrast 0.21 .

The right column of Figure 2 displays the information for the finger rotated about its axis by $+20^{\circ}$ (direction of rotation equivalent to pronation). As seen in the top row, rotation of the finger caused the receptive field center to move, and initial contact with the grating was lateral to this point. Indenting the finger into the grating by $1 \mathrm{~mm}$ produced a contact oval (second and third row) such that the receptive field center was located not in the middle of the oval, but off to the ulnar side of it. The cycle histogram (bottom row) is still asymmetric, with the SA still responding less vigorously to grating movement in the ulnar direction. The ratio of the mean responses for the 2 half cycles was 1.5 and the contrast was 0.21 . Similarly, the left column of Figure 2 displays the results when the finger was rotated about its axis by $-20^{\circ}$ (direction of rotation equivalent to supination). In this case, the contact oval was such that the receptive field center was located off to the radial side of it. The cycle histogram is now close to symmetric, with the response still having a marginal preference for grating movement in the radial direction (ratio 1.2, contrast 0.07 ). The contact ovals in Figure 2 were estimated by fingerprinting as described in Materials and Methods.

In Figure $3 A$ the responses of the afferent from Figure 2 are shown as a function of the angle of rotation of the finger over a range from $-35^{\circ}$ to $+35^{\circ}$. Even though the center of this afferent's receptive field was estimated to lie at the center of the fingerpad, the response at $0^{\circ}$ was obviously direction selective. Moreover, when the finger was rotated by up to $-35^{\circ}$ and by up to $+35^{\circ}$, the responses were still asymmetric, with the same preferred (radial) direction. As seen at the bottom of Figurc $3 A$, these rotations moved the center of the receptive field from one edge of the contact oval to the opposite edge of the oval.

Figure $3 B$ shows the responses of a second $\mathrm{SA}$ whose receptive field center was also estimated to be located at the center of the fingerpad. The responses of this afferent were essentially symmetric (i.e., not direction selective) over the complete range of rotation from $-40^{\circ}$ to $+40^{\circ}$. As shown at the bottom of Figure $3 B$, this range of rotation moved the center of the receptive field 


\begin{tabular}{|c|c|c|c|c|c|c|c|}
\hline \multirow{2}{*}{$\begin{array}{l}\text { Fre- } \\
\text { quency }\end{array}$} & \multicolumn{7}{|c|}{ Spatial period $(\mathrm{mm})$} \\
\hline & 0.75 & 1.0 & 1.25 & 1.5 & 1.75 & 2.0 & 3.0 \\
\hline \multicolumn{8}{|l|}{$10 \mathrm{~Hz}$} \\
\hline$\mu$ & & & & & & & 0.123 \\
\hline$\sigma$ & & & & & & & 0.146 \\
\hline$n$ & & & & & & & 3 \\
\hline \multicolumn{8}{|l|}{$20 \mathrm{~Hz}$} \\
\hline$\mu$ & & & & 0.251 & 0.485 & 0.206 & 0.164 \\
\hline$\sigma$ & & & & 0.324 & & 0.309 & 0.169 \\
\hline$n$ & & & & 3 & 2 & 6 & 6 \\
\hline \multicolumn{8}{|l|}{$40 \mathrm{~Hz}$} \\
\hline$\mu$ & 0.173 & 0.143 & 0.235 & 0.120 & 0.279 & 0.196 & 0.155 \\
\hline$\sigma$ & 0.139 & 0.162 & 0.265 & 0.132 & & 0.284 & 0.094 \\
\hline$n$ & 4 & 7 & 3 & 7 & 2 & 6 & 7 \\
\hline \multicolumn{8}{|l|}{$80 \mathrm{~Hz}$} \\
\hline$\mu$ & 0.079 & 0.118 & 0.171 & 0.060 & 0.158 & 0.072 & 0.097 \\
\hline$\sigma$ & 0.053 & 0.170 & 0.226 & 0.052 & & 0.075 & 0.077 \\
\hline$n$ & 7 & 13 & 3 & 7 & 2 & 12 & 7 \\
\hline \multicolumn{8}{|l|}{$160 \mathrm{~Hz}$} \\
\hline$\mu$ & 0.138 & 0.074 & 0.090 & 0.114 & 0.143 & 0.059 & 0.058 \\
\hline$\sigma$ & 0.106 & 0.027 & 0.087 & 0.123 & & 0.040 & 0.022 \\
\hline$n$ & 7 & 7 & 3 & 7 & 2 & 7 & 7 \\
\hline \multicolumn{8}{|l|}{$320 \mathrm{~Hz}$} \\
\hline$\mu$ & 0.096 & 0.098 & 0.060 & 0.128 & & & \\
\hline$\sigma$ & 0.103 & 0.086 & 0.058 & 0.027 & & & \\
\hline$n$ & 7 & 6 & 3 & 7 & & & \\
\hline
\end{tabular}

Format as in Table 1.

from outside the contact oval on one side to outside the contact oval on the opposite side.

As seen at the top of Figure $3 C$, the receptive field of the third SA illustrated was located on the central portion of the fingerpad but to the radial side of the midline. At $0^{\circ}$ the response of the afferent was not direction selective. At rotations of $+10^{\circ}$ and $+20^{\circ}$ the response was direction selective, being greater for movement in the ulnar direction, while for rotations of $-20^{\circ}$ and $-40^{\circ}$ the response was selective for movement in the radial direction.

Classifying the nature of the direction selectivity of the afferent responses is not simple. In order to assess this we systematically performed a rotation analysis, as illustrated for the afferents in Figures 2 and 3, on 13 SAs, 13 RAs, and 4 PCs. For most afferents the experiments were performed for 2 combinations of the 3 stimulus parameters, namely, the grating spatial periods, peak movement speeds, and peak temporal frequencies of the ridges. For SAs these parameters were $3 \mathrm{~mm}, 60 \mathrm{~mm} / \mathrm{sec}, 20$ $\mathrm{Hz}$ and $2 \mathrm{~mm}, 40 \mathrm{~mm} / \mathrm{sec}, 20 \mathrm{~Hz}$; for RAs, $2 \mathrm{~mm}, 80 \mathrm{~mm} / \mathrm{sec}$, $40 \mathrm{~Hz}$ and $1 \mathrm{~mm}, 40 \mathrm{~mm} / \mathrm{sec}, 40 \mathrm{~Hz}$; and for PCs, $2 \mathrm{~mm}, 160$ $\mathrm{mm} / \mathrm{sec}, 80 \mathrm{~Hz}$ and $1 \mathrm{~mm}, 80 \mathrm{~mm} / \mathrm{sec}, 80 \mathrm{~Hz}$. For any particular afferent, the curves of response versus rotation angle were not identical for the 2 parameter combinations used. However, they were very similar and showed the same response trends. For descriptive purposes we have classified the responses into 3 groups, namely, direction selective (like the afferent illustrated in Fig. $3 A$ ), not direction selective (like the afferent illustrated in Fig. $3 B$ ), and reversing direction selective (like the afferent illustrated in Fig. 3C).
Among the SAs, about $60 \%$ of the responses were direction selective and about $30 \%$ were not. There seemed to be no relationship between the nature of the response and the position of the receptive field center, either in regard to the presence or absence of direction selectivity or to the direction of movement eliciting the greater response when selectivity was present. Only a single SA exhibited reversing direction sclectivity (the afferent in Fig. 3C). In this case, the greatest response occurred when the direction of grating movement was "towards" the receptive field center.

Similarly among the RAs, about $60 \%$ of the responses were direction selective and about $30 \%$ were not. Again, there appeared to be no relationship between the nature of the response and the position of the receptive field center. Two RAs exhibited reversing direction selectivity: for one the direction of maximum response was "towards" the receptive field center and for the other "away from" the receptive field center.

The 4 PCs used in the rotation study were selected because their receptive fields could be localized with some confidence. The receptive field center for one afferent was located in the middle of the fingerpad and for a second afferent around the distal interphalangeal joint. For neither of these afferents were the responses direction selective. The third PC's receptive field center was located on the radial side of the fingerpad. For the $2 \mathrm{~mm}$ surface, the response was not direction selective, while for the $1 \mathrm{~mm}$ surface, the response was greater for movement in the ulnar direction. The fourth PC's receptive field center was located on the ulnar side of the fingerpad. For the $1 \mathrm{~mm}$ surface, the response was greatest for movement in the ulnar direction, 
A.

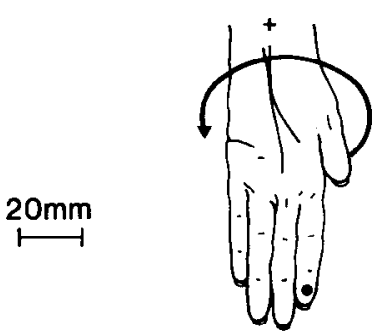

B.

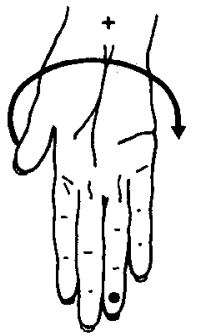

C.

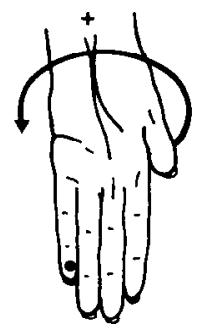

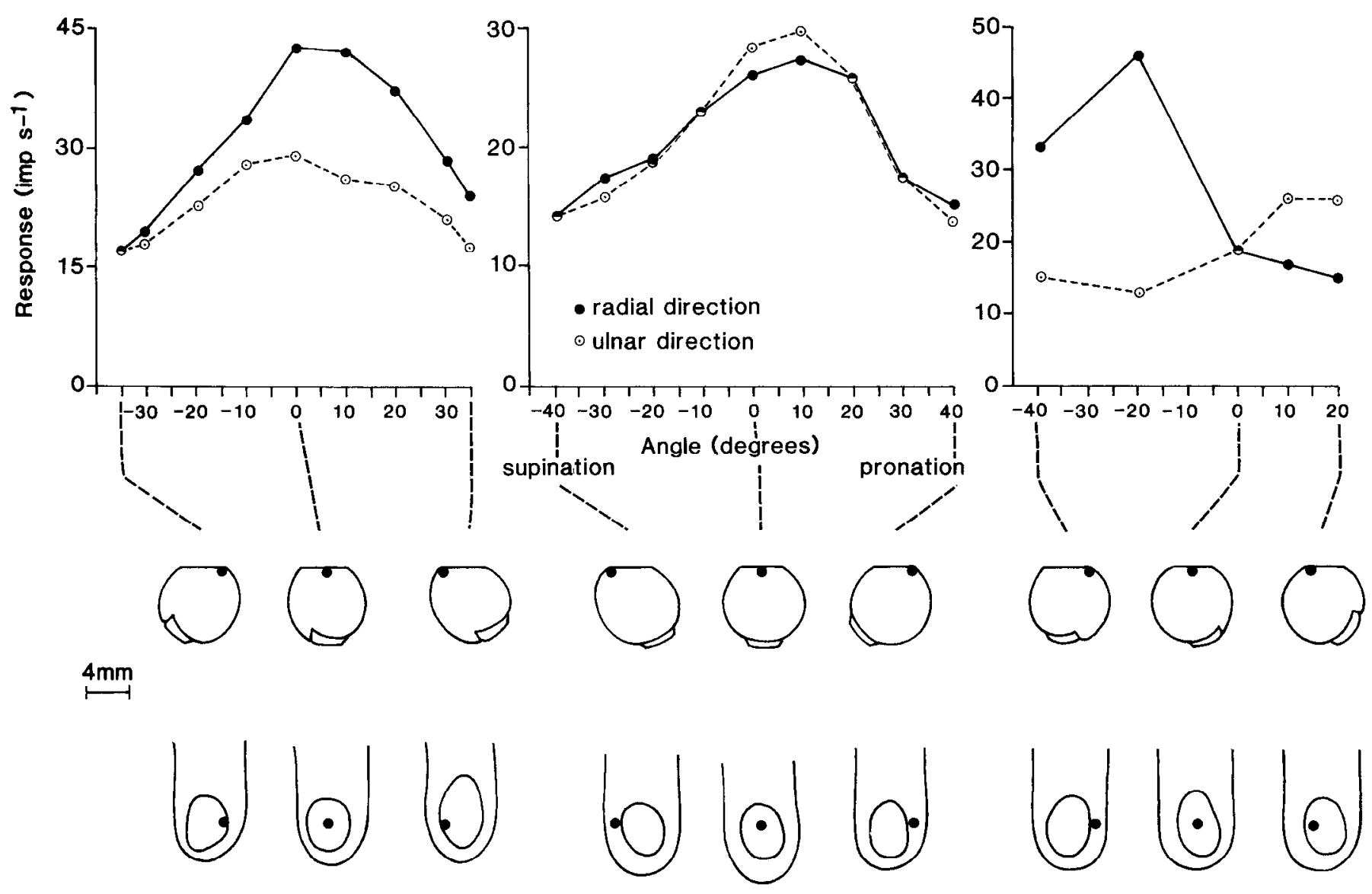

Figure 3. Assessment of response symmetry in 3 different SAs (columns $A, B$, and $C$, respectively). In the top row, each dot shows the position of the receptive field center (RFC), and arrows indicate the direction of pronation. In the second row, the mean responses over the 2 half cycles of movement are plotted as a function of the angle of rotation of the finger about its axis. The solid line and filled circles show responses during the half cycle when movement was in the radial direction and the broken line and open circles when movement was in the ulnar direction. Angles are denoted positive or negative when the direction of rotation corresponded to pronation or supination, respectively. The third row shows an end view of the finger and the relationship of the RFC (dot) to the contact area when the finger was indented $1 \mathrm{~mm}$ into the grating. The bottom row shows a top (ventral) view of the finger and the relationship of the RFC to the contact oval. For $A, B$, and $C$ the spatial period of the grating was $3 \mathrm{~mm}$, the peak speed of movement $60 \mathrm{~mm} / \mathrm{sec}$, and the peak temporal frequency of the ridges $20 \mathrm{~Hz}$.

while for the $2 \mathrm{~mm}$ surface, the direction selectivity reversed with the maximum response occurring with movement "towards" the receptive field center.

\section{Changing indentation amplitude}

In our experiments, contact between the skin and the grating was controlled by the amplitude of indentation of the fingertip into the grating from the point of contact. For all the data described so far, this value was $1 \mathrm{~mm}$. The following experiments describe the effects of changing indentation amplitude on the responses of the afferent fibers.

For the SA afferent illustrated in Figure 4, a grating with a spatial period of $3 \mathrm{~mm}$ was moved sinusoidally over the receptive field with a peak speed of $60 \mathrm{~mm} / \mathrm{sec}$, and the peak temporal frequency of the ridges was thus $20 \mathrm{~Hz}$. Four amplitudes of indentation were used, namely, $0.5,0.75,1$, and $1.25 \mathrm{~mm}$, and 
the effect on the responsc of the afferent was characterized in a block of data gathered in the following temporal sequence: (1) Data were collected for a run of 16 cycles of movement at an indentation amplitude of $0.5 \mathrm{~mm}$; (2) the finger was removed from the grating for a period of at least $1 \mathrm{~min}$; (3) the process was repeated for runs of 16 cycles at indentation amplitudes of $0.75 \mathrm{~mm}$, then $1 \mathrm{~mm}$, and then $1.25 \mathrm{~mm}$ with the finger removed from the grating for at least $1 \mathrm{~min}$ between runs. To assess the repeatability of the observations, this entire block was performed 6 times for this afferent.

As seen in Figure 4 (top), the mean response of the SA, averaged over a cycle of movement, increased as the indentation amplitude increased. The small SEs indicate the highly repeatable nature of the observation. The relationship can be adequately characterized by a straight line (correlation coefficient $r=0.992$ ). As seen in Figure 4 (bottom), the mean contact force, averaged over a cycle of movement, increased monotonically as the indentation amplitude increased: the relationship $(r=$ 0.960 ) was not as linear as the response indentation relationship, with the rate of increase in force also increasing as the indentation amplitude increased.

As seen in Figure 4 (center), increasing the indentation amplitude also increased the contact oval between the finger and the grating (these were estimaled by fingerprinting as outlined in Materials and Methods). It has been suggested that the appropriate index of contact is neither the indentation amplitude nor the contact force, but rather the contact pressure (Morley et al., 1983). This was estimated by dividing the measured contact force by the estimated area of the contact oval, as shown at the bottom of Figure 4. When the amplitude of indentation increased, the contact force and contact area increased concomitantly; as a result, contact pressure did not vary as much. Thus, for the SA in Figure 4, mean response increased approximately linearly with indentation amplitude $(r=0.992)$ or with contact force $(r=0.966)$ but was much less dependent on contact pressure $(r=0.803)$.

We compared the responses of many SA afferents to indentation amplitudes of 1 and $1.25 \mathrm{~mm}$ over a wide range of grating spatial periods and peak movement speeds. In all cases the mean response, averaged over a cycle of movement, was greater for the greater indentation amplitude. To quantify this effect, 10 SAs were tested at indentation amplitudes of $0.5,0.75,1$, and $1.25 \mathrm{~mm}$ using 2 gratings, namely, a $3 \mathrm{~mm}$ grating moving at a peak speed of $60 \mathrm{~mm} / \mathrm{sec}$ and a $2 \mathrm{~mm}$ grating moving at a peak speed of $40 \mathrm{~mm} / \mathrm{sec}$ (in both cases the peak temporal frequency of ridges was $20 \mathrm{~Hz}$ ). All the SAs exhibited the same behavior, with the response consistently increasing monotonically with an increase in indentation amplitude, for both gratings.

Different SAs have different sensitivities, so that, for a given combination of stimulus parameters, the response magnitude varied widely from SA to SA. Therefore, to quantify the relationship between indentation amplitude and response, the sensitivity of the individual fibers was eliminated by normalizing prior to combining the data for the 10 afferents. For each afferent the responscs were divided by the sum of the responses at the 4 indentation amplitudes. The sum was chosen, rather than a single common response (e.g., the response at $1.25 \mathrm{~mm}$ ), as this normalizing factor is less sensitive to errors in individual responses. Figure 5 (left) shows the pooled, normalized responses of the 10 fibers. For clarity, the SEs are shown only on the 3 $\mathrm{mm}$ curve: they are very similar for the $2 \mathrm{~mm}$ curve and details
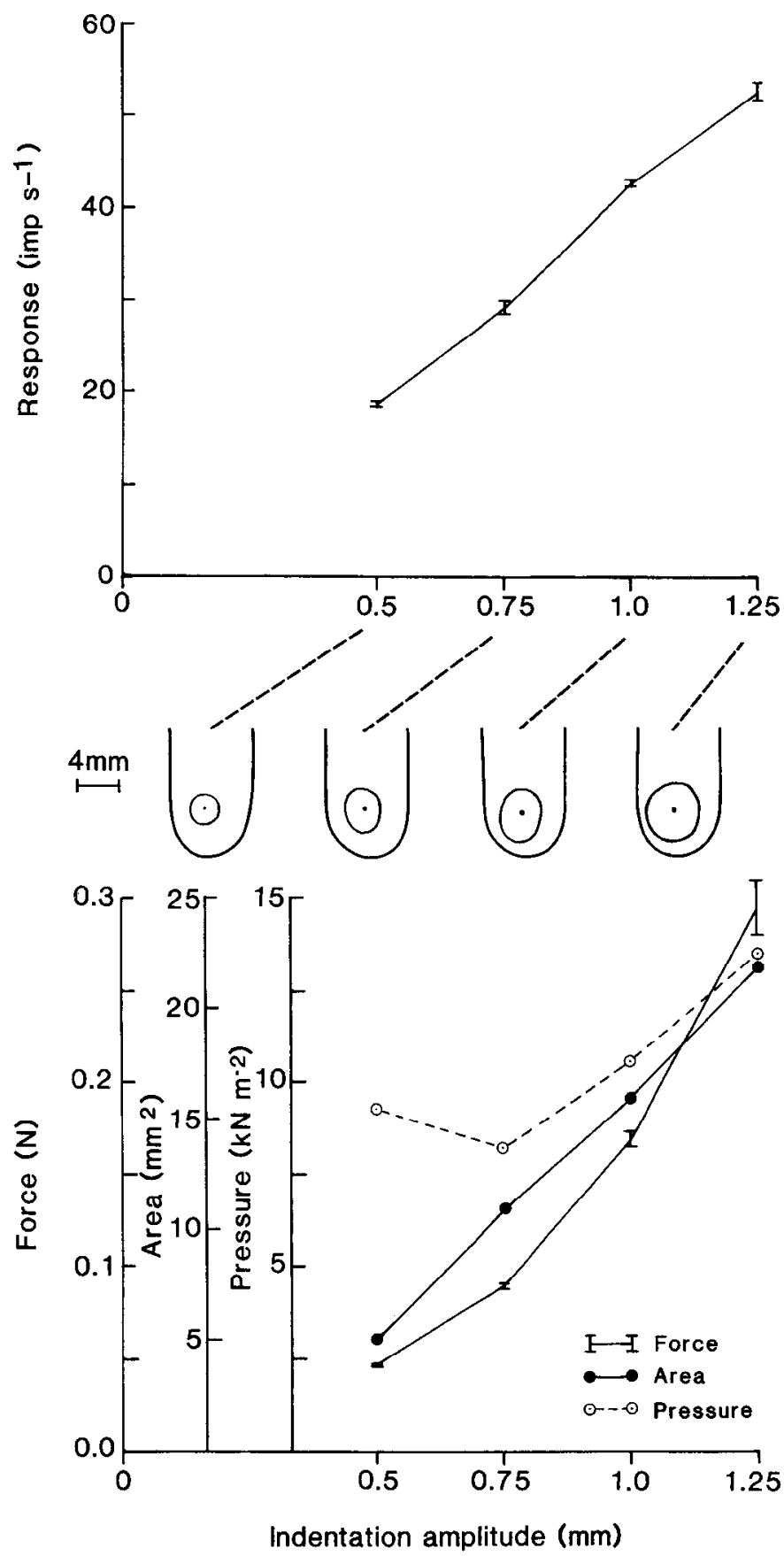

Figure 4. Responses of an SA to changes in indentation amplitude. $T o p$, Mean cyclic responses with error bars indicating \pm 1 SEM $(n=6)$. Middle, Ventral (top) view of the finger showing the contact ovals and the center of the receptive field (dot). Bottom, Solid line with error bars $( \pm 1$ SEM, $n=6)$ shows contact force; solid line with filled circles shows area of contact oval; broken line with open circles shows contact pressure.

are given in the legend. For both the gratings ( 3 and $2 \mathrm{~mm}$ spatial periods), the response obviously increased monotonically as the indentation amplitude increased. However, for the $3 \mathrm{~mm}$ grating the relationship was more linear (correlation coefficient $r=0.957$, slope $=0.296)$ than for the $2 \mathrm{~mm}$ grating $(r=$ 0.761 , slope $=0.204)$. The major difference is that for the 2 $\mathrm{mm}$ grating, increasing the indentation amplitude from 0.5 to $0.75 \mathrm{~mm}$ had less effect than an increase from 1 to $1.25 \mathrm{~mm}$. In addition to the above data, we have available the responses 

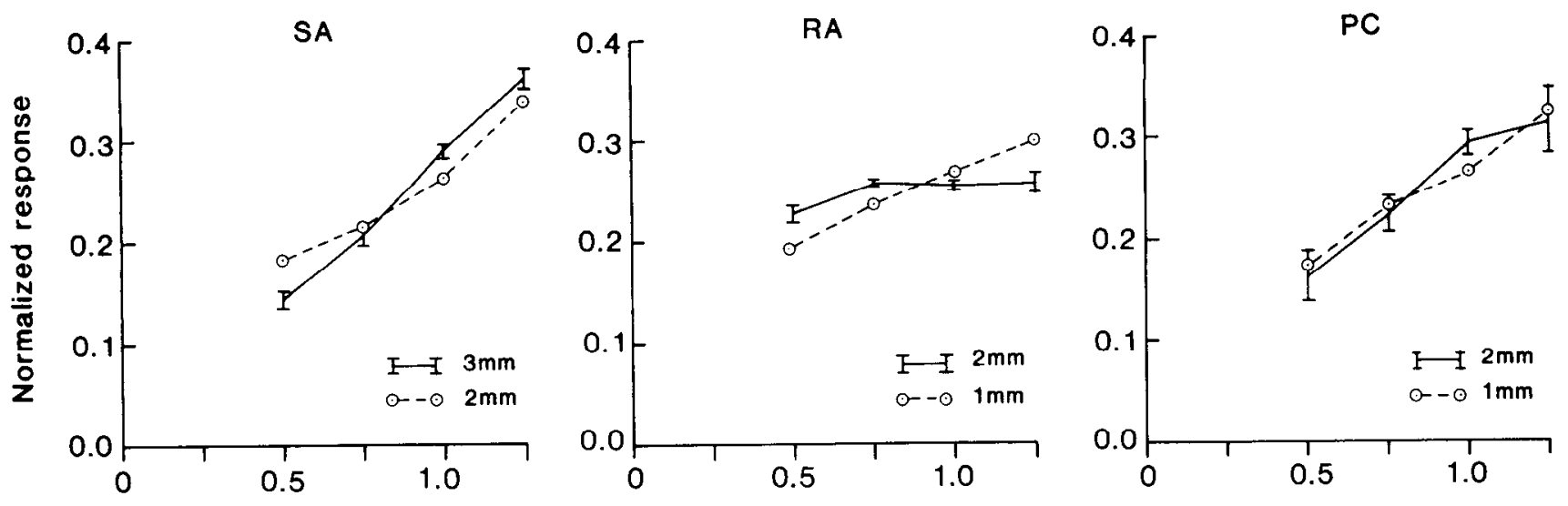

Indentation amplitude ( $\mathrm{mm})$

Figure 5. Mean responses of the SA, RA, and PC populations as a function of indentation amplitude. To eliminate variability caused by the sensitivity of individual afferents, responses were normalized before combining. For the SAs, responses are shown for 3 and 2 mm gratings moving at peak speeds of 60 and $40 \mathrm{~mm} / \mathrm{sec}$, respectively (peak temporal frequency of the ridges, $20 \mathrm{~Hz}$ ). The SEs at $0.5,0.75,1$, and $1.25 \mathrm{~mm}$ were 0.008 , $0.008,0.007$, and 0.010 for the $3 \mathrm{~mm}$ grating $(n=10)$ and $0.019,0.013,0.008$, and 0.023 for the $2 \mathrm{~mm}$ graling $(n=9)$, respectively. For the RAs, responses are shown for 2 and $1 \mathrm{~mm}$ gratings moving at peak speeds of 80 and $40 \mathrm{~mm} / \mathrm{sec}$ respectively (peak temporal frequency of the ridges, 40 $\mathrm{Hz}$ ). The SEs at $0.5,0.75,1$, and $1.25 \mathrm{~mm}$ were $0.008,0.003,0.006$, and 0.009 for the $2 \mathrm{~mm}$ grating $(n=9)$ and $0.019,0.005,0.009$, and 0.010 for the $1 \mathrm{~mm}$ grating $(n=8)$, respectively. For the PCs, responses are shown for 2 and $1 \mathrm{~mm}$ gratings moving at peak speeds of 160 and 80 mm/ $\mathrm{sec}$, respectively (peak temporal frequency of the ridges, $80 \mathrm{~Hz}$ ). The SEs at $0.5,0.75,1$, and $1.25 \mathrm{~mm}$ were $0.026,0.017,0.012$ and 0.033 for the $2 \mathrm{~mm}$ grating $(n=5)$ and $0.035,0.015,0.008$, and 0.046 for the $1 \mathrm{~mm}$ grating $(n=5)$, respectively. For clarity, the SEs are shown on only 1 curve for each afferent type.

of $18 \mathrm{SAs}$ to indentation amplitudes of 1 and $1.25 \mathrm{~mm}$. For the $3 \mathrm{~mm}$ grating the ratio of responses at indentation amplitudes of 1.25 and $1 \mathrm{~mm}$, respectively, had a mean value of 1.27 (SE 0.041 ). For the $2 \mathrm{~mm}$ grating the ratio had a mean value of 1.31 (SE 0.057). These statistics on the larger pool of 18 SAs are entirely consistent with the smaller pool of 10 SAs in Figure 5.

Increasing the indentation amplitude from 1 to $1.25 \mathrm{~mm}$, over a range of grating spatial periods and peak movement speeds, had an inconsistent effect on the responses of the RA afferents. To characterize the RA population, responses were measured in 9 fibers to indentation amplitudes of $0.5,0.75,1$, and $1.25 \mathrm{~mm}$. Two gratings were used, namely, a grating with a spatial period of $2 \mathrm{~mm}$ moving at a peak speed of $80 \mathrm{~mm} / \mathrm{sec}$ and a grating with a spatial period of $1 \mathrm{~mm}$ moving at a peak speed of $40 \mathrm{~mm} / \mathrm{sec}$ (peak temporal frequency of ridges was 40 $\mathrm{Hz}$ in both cases). For the $1 \mathrm{~mm}$ grating, the response increased monotonically as a function of indentation amplitude for all 9 afferents. For the $2 \mathrm{~mm}$ grating, an increase in indentation amplitude sometimes caused an increase in response, sometimes a decrease, and sometimes no change at all. Before pooling the data (Fig. 5, center), the sensitivity of the individual RAs was eliminated by normalizing responses by dividing by the sum of responses at the 4 indentation amplitudes (as for the SAs). In the case of the $1 \mathrm{~mm}$ grating, the response clearly increased monotonically with increasing indentation amplitude. The relationship was fairly linear $(r=0.772$, slope $=0.138)$, but the sensitivity or slope was much less than for the SAs (Fig. 5, left). For the $2 \mathrm{~mm}$ grating the relationship was not monotonic $(r=$ 0.419 ). On increasing the indentation amplitude from 0.5 to $0.75 \mathrm{~mm}$ the response incrcascd, but further increases in indentation amplitude had little or no significant effect.

The effect of increasing indentation amplitude was studied on 5 PCs. All had receptive fields that could be localized, with some confidence, to the distal phalanx. Two gratings were used with spatial periods of 2 and $1 \mathrm{~mm}$ moving at peak speeds of 160 and $80 \mathrm{~mm} / \mathrm{sec}$, respectively, both resulting in a peak tem- poral frequency of the ridges of $80 \mathrm{~Hz}$. For both gratings the 5 afferents showed consistent behavior, with an increase in indentation amplitude causing an increase in the mean response averaged over a cycle of movement. As for the SAs and RAs, the sensitivity of individual PCs was eliminated by normalizing prior to pooling the data in Figure 5 (right). For both surfaces the relationship was monotonic. Linear regression yields a correlation coefficient of 0.777 and a slope of 0.212 for the $2 \mathrm{~mm}$ grating and a correlation coefficient of 0.666 and a slope of 0.193 for the $1 \mathrm{~mm}$ grating.

Comparing the 3 sets of curves in Figure 5, indentation amplitude had the greatest effect on the SA afferents when stimulated with a $3 \mathrm{~mm}$ grating, slightly less effect on the SA afferents stimulatcd with a $2 \mathrm{~mm}$ grating or on the PC afferents stimulated with a 1 or $2 \mathrm{~mm}$ grating, and still less effect on the RA afferents stimulated with a $1 \mathrm{~mm}$ grating. When stimulated with a $2 \mathrm{~mm}$ grating, the RA responses did not increase monotonically.

\section{Discussion}

\section{Asymmetry of responses}

In the visual system, direction-selective cells are common in the visual cortex and also occur among retinal ganglion cells (Peterhans et al., 1985). Such neurons respond maximally when a visual target moves in one direction and respond less vigorously or not at all when the target moves in the opposite direction. The visual receptors are presumed to have symmetric responses, and the direction selectivity of neurons further along the visual pathway is attributed to the particulars of the synaptic connections. A common model is one in which inhibition precedes excitation when the target moves in the nonpreferred direction, reducing or eliminating the response (Barlow and Levick, 1965). In the tactile system, direction-selective units have been found in the postcentral gyrus (Schwartz and Fredrickson, 1971; Whitsel et al., 1972; Hyvärinen and Poranen, 1978; Iwamura et al., 1985). These respond optimally when tactile stimuli 
move over the skin in a particular direction. Here, too, inhibitory synaptic connections have been postulated to explain the direction selectivity (Gardner and Costanzo, 1980), and there is an assumption, either explicit or implicit, that the responses at the primary afferent level are symmetric.

In our experiments, asymmetric responses occurred in all 3 mechanoreceptive afferent groups (SAs, RAs, and PCs); in fact, they were more common than completely symmetric responses. In retrospect, this is perhaps not surprising. The only structures hetween visual receptors and visual targets are those forming the optics of the eye, and these have isotropic light transmission properties. In contrast, tactile receptors are excited by stresses and strains, in the environment of their membranes, produced by interaction between the moving stimulus and the complex mechanics of the skin. Skin is not isotropic (Tregear, 1966), and even at a simple level there are obvious factors that could lead to asymmetric responses. For example, in typical cross sections of fingertip skin (Halata, 1975) the dermal papillae are not all precisely orthogonal to the skin surface, so that the effective stimulus at a Meissner corpuscle located at the crest of a papilla may well be different for opposite directions of movement of a grating over the skin. Moreover, the corpuscle is connected to the dermis and epidermis through an array of collagen fibers (Andres and von Düring, 1973), and it is possible that the efficacy of these connections is not symmetric. A similar argument holds for the Merkel cell-neurite complex, the Ruffini ending, and even for the PC corpuscle, although its greater depth in the skin may suggest that asymmetries in the epidermis or dermis would be less important. In the case of Meissner corpuscles and Merkel complexes, multiple receptors are connected to each afferent fiber (Sinclair, 1981). Thus, it is possible that interaction between receptors produces some asymmetry in the afferent's responses. However, we have no model for such an interaction.

When humans rub their fingertips over a grating, a population of SAs and RAs innervating the region of skin forming the contact oval will be active. The majority of these afferents will respond asymmetrically. There will be an additional population of active SAs and RAs, with receptive field centers outside the contact oval, that also respond asymmetrically. Our data show that these afferents will display a spectrum of responses ranging from a greater response to a particular direction of movement, through equal responses in both directions, to a greater response to the second direction of movement. We were unable to predict the nature of an individual response from the position of the afferent's receptive field center with respect to the contact oval. In the case of the PCs, most of the corpuscles, located anywhere in the finger, will be active. The majority will be located to one side of the fingerpad or will be associated with an interphalangeal joint or perhaps a more proximal phalanx of the finger; few, if any, could be stated to have a receptive field center closely corresponding to the contact oval. Nevertheless, this responding population conveys information about the grating, and here, too, the responses span a range of asymmetries.

When a monkey actively scans its fingers over a grating, many of the cells in areas $3 b$ and 1 of the postcentral gyrus have asymmetric responses (Darian-Smith et al., 1984). One possible explanation given for these asymmetries, in effect, is that the receptive field center was located to one side of the contact oval and thus was more effectively engaged for one direction of movement than for the other. Our data show that asymmetry in response at the primary afferent level occurs not only when the receptive field centers are located outside the contact oval, but also when they are located at the center of the contact oval, and this may account for at least some of the cortical asymmetry. The completely direction-selective units seen in the cortex, especially those with large receptive fields such as occur in area 2 (Costanzo and Gardner, 1980; Darian-Smith et al., 1984), are undoubtedly a product of cortical circuitry. With active movement of the finger over the grating there is the additional problem of rotation of the finger so that the contact oval itself moves during the cycle of movement.

\section{Indentation amplitude and contact force}

The majority of quantitative studies of mechanoreceptors in the glabrous skin have utilized punctate probes indenting into the skin. Here, the magnitude of the stimulus may be taken either as the amplitude of indentation or as the contact force between the skin and the probe. These 2 parameters are of course related, under certain circumstances linearly (Pubols, 1982). In the case of a grating moving across the skin, the amplitude of indentation and the contact force are related as shown in Figure 4. With a large surface, such as a grating, the contact area also increases with increasing indentation amplitude or contact force. Thus, contact pressure is a third measure of contact and is often used as the basis for selecting experimental parameters.

The mean cyclic responses of the SAs and PCs increascd with increasing indentation amplitude for both surfaces tested. The RA afferents showed similar behavior in response to a grating with a spatial period of $1 \mathrm{~mm}$, but their response to a $2 \mathrm{~mm}$ grating did not increase with increasing indentation amplitude. The mean cyclic response correlated about equally well with the indentation amplitude and with the contact force, but there was a poorer correlation with the contact pressure. These results may explain Lederman and Taylor's (1972) observation that, in the human, the perceived roughness of gratings increases with increasing contact force. Human discrimination of textured surfaces occurs successfully over a wide range of contact forces (Lamb, 1983). This does not eliminate the use, by the subject, of force-dependent parameters like mean cyclic response, as long as the same force conditions are used within a pair of surfaces being discriminated.

Previous studies have suggested that even a large increase in contact force has little or no effect on the responses of any of the afferents (Darian-Smith and Oke, 1980). This is not consistent with our data and presumably occurred because these authors did not study the effects of contact force systematicallythey were primarily interested in factors in the response other than mean response. For instance, when the indentation amplitude is changed, the response remains phase-locked over the central $42^{\circ}$ of the cycle (Morley and Goodwin, 1987), but of course the number of impulses per grating ridge changes.

The effective stimulus for a mechanoreceptor is dependent on the stresses and strains in the environment of the receptor. How these relate to parameters like indentation amplitude or contact force depends on the complex nature of the intervening skin. As we have no comprehensive model for skin mechanics, we cannot explain our observations at this level. It is intuitively obvious that increasing indentation amplitude will increase some stresses and strains in the skin, so our results are not surprising.

\section{References}

Andres, K. H., and M. von Düring (1973) Morphology of cutaneous receptors. In Handbook of Sensory Physiology, Vol. 2, A. Iggo, ed., pp. 1-28, Springer-Verlag, Berlin. 
Barlow, H. B., and W. R. Levick (1965) The mechanism of directionally selective units in rabbit's retina. J. Physiol. (Lond.) 178:477504.

Costanzo, R. M., and E. P. Gardner (1980) A quantitative analysis of responses of direction-sensitive neurons in somatosensory cortex of awake monkeys. J. Neurophysiol. 43: 1319-1341.

Darian-Smith, I., and L. E. Oke (1980) Peripheral neural representation of the spatial frequency of a grating moving across the monkey's finger pad. J. Physiol. (Lond.) 309: 117-133.

Darian-Smith, I., I. Davidson, and K. O. Johnson (1980) Peripheral neural representation of spatial dimensions of a textured surface moving across the monkey's finger pad. J. Physiol. (Lond.) 309: 135-146.

Darian-Smith, I., A. Goodwin, M. Sugitani, and J. Heywood (1984) The tangible features of textured surfaces: Their representation in the monkey's somatosensory cortex. In Dynamic Aspects of Neocortical Function, G. W. Edelman, W. E. Gall, and W. M. Cowan, eds., pp. 475-500, Wiley, New York.

Gardner, E. P., and R. M. Costanzo (1980) Neuronal mechanisms underlying direction sensitivity of somatosensory cortical neurons in awake monkeys. J. Neurophysiol. 43: 1342-1354.

Goodwin, A. W, and J. W. Morley (1987) Sinusoidal movement of a grating across the monkey's fingerpad: Representation of grating and movement features in afferent fiber responses. J. Neurosci., 7:21682180.

Halata, Z. (1975) The mechanoreceptors of the mammalian skin ultrastructure and morphological classification. Adv. Anat. Embryol. Cell Biol. 50: 1-77.

Hyvärinen, J., and A. Poranen (1978) Movement-sensitive and direction and orientation-selective cutaneous receptive fields in the hand area of the post-central gyrus in monkeys. J. Physiol. (Lond.) 283: 523-537.

Iwamura, Y., M. Tanaka, M. Sakamoto, and O. Hikosaka (1985) Functional surface integration, submodality convergence, and tactile feature detection in Area 2 of the monkey somatosensory cortex. In Hand Function and the Neocortex, A. W. Goodwin and I. DarianSmith, eds., pp. 44-58, Springer-Verlag, Heidelberg.

Johnson, K. O. (1974) Reconstruction of population response to a vibratory stimulus in quickly adapting mechanoreceptive afferent fiber population innervating glabrous skin of the monkey. J. Neurophysiol. 35: 48-72.
Johnson, K. O., and G. D. Lamb (1981) Neural mechanisms of spatial tactile discrimination: Neural patterns evoked by braille-like dot patterns in the monkey. J. Physiol. (Lond.) 310: 117-144.

Knibestöl, M. (1975) Stimulus-response functions of slowly adapting mechanoreceptors in the human glabrous skin area. J. Physiol. (Lond.) 245: 63-80.

Lamb, G. D. (1983) Tactile discrimination of textured surfaces: Psychophysical performance measurements in humans. J. Physiol. (Lond.) $338: 551-565$.

Lederman, S. J., and M. M. Taylor (1972) Fingertip force, surface geometry, and the perception of roughness by active touch. Percept. Psychophys. 12: 401-408.

Lindblom, U., and L. Lund (1966) The discharge from vibrationsensitive receptors in the monkey foot. Exp. Neurol. 15: 401-417.

Morley, J. W., and A. W. Goodwin (1987) Sinusoidal movement of a grating across the monkey's fingerpad: Temporal patterns of afferent fiber responses. J. Neurosci., 7: 2181-2191.

Morley, J. W., A. W. Goodwin, and I. Darian-Smith (1983) Tactile discrimination of gratings. Exp. Brain Res. 49: 291-299.

Mountcastle, V. B., W. H. Talbot, and H. H. Kornhuber (1966) 'T he neural transformation of mechanical stimuli delivered to the monkey's hand. In Touch, Heat and Pain, A. V. S. De Reuck and K. Knight, eds., pp. 325-345, Ciba Foundation, Churchill, London.

Peterhans, E., P. O. Bishop, and R. M. Camarda (1985) Direction selectivity of simple cells in cat striate cortex to moving light bars. I. Relation to stationary flashing bar and moving edge responses. Exp. Brain Res. 57: 512-522.

Pubols, B. H., Jr. (1982) Factors affecting cutaneous mechanoreceptor response. I. Constant-force versus constant-displacement stimulation. J. Neurophysiol. 47: 515-529.

Schwartz, D. W. F., and J. M. Fredrickson (1971) Tactile direction sensitivity of area 2 oral neurons in the rhesus monkey cortex. Brain Res. 27: 391-401.

Sinclair, D. (1981) Mechanisms of Cutaneous Sensation, Oxford U. P., New York.

Tregear, R. T. (1966) Physical Functions of Skin, Academic, London.

Whitsel, B. L., J. R. Roppolo, and G. Werner (1972) Cortical information processing of stimulus motion on primate skin. J. Neurophysiol. 35: 691-717. 